\title{
Synthesis and Characterization of Multifunctional Chiral and Photoactive Organic-Inorganic Block Copolymers of Poly(methylphenylsilane) with (R)-N-(1-phenylethyl)methacrylamide, Disperse Red 1 Methacrylate and Their optical and Photophysical Properties
}

\author{
Km. Meenu ${ }^{a}$, Dibyendu S. Bag ${ }^{b}$, Rekha Lagarkhac ${ }^{c}$, Radha Tomard and Arvind Kumar \\ Gupta $^{\mathbf{e}}$
}

(a, b): Defence Material and Stores Research and Development Establishment (DMSRDE) DMSRDE P. O., G. T. Road, Kanpur-208013, INDIA

(c): Department of Chemistry Bundelkhand University Jhansi-284128, INDIA (d): SOS in Chemistry, Jiwaji University, Gwalior-474011, INDIA

(e): Defence Research and Development Establishment (DRDE), Gwalior-474001, INDIA $\underline{\text { singhmeenu} 458 @ \text { gmail.com }}$

\begin{abstract}
Multifunctional polymers of poly(methylphenylsilane) (PMPS) with (R)-N-(1phenylethyl)methacrylamide (R-NPEMAM) and disperse red 1 methacrylate (DR1MA) were synthesized in a quartz tube using UV-technique. The molecular weights of such synthesized polymers were of the order of $10^{3}$. The appearance of two glass transition temperatures in DSC indicated the synthesized polymers as block copolymers. The optical absorbance was observed at $272 \mathrm{~nm}, 327 \mathrm{~nm}$ and $475 \mathrm{~nm}$ due to $\pi-\pi^{*}, \sigma-\sigma^{*}$ and $\mathrm{n}-\pi^{*}$ with $\pi-\pi^{*}$ transition respectively. The circular dichroism observed at $261 \mathrm{~nm}$ due to the chiral unit present in the polymeric chain. They also exhibited induced chirality at $330 \mathrm{~nm}$ and $470 \mathrm{~nm}$ due to associated with the Si-Si chain of PMPS and azobenzene chromophore of DR1MA unit, respectively. In photoluminescence properties two photoemissions $\left(\lambda_{\mathrm{em}}\right)$ were observed at 307 $\mathrm{nm}$ and $415 \mathrm{~nm}$ when excited at $275 \mathrm{~nm}$. The $\lambda_{\mathrm{em}}$ was also observed at $415 \mathrm{~nm}$ when excited by $325 \mathrm{~nm}$. The multiemission spectra were observed at $500 \mathrm{~nm}, 550 \mathrm{~nm}$ and $590 \mathrm{~nm}$ when excited by $475 \mathrm{~nm}$ which may be due to exciton coupling between azobenzene chromophore and $\mathrm{Si}$-Si $\sigma$-conjugation. Such multifunctional polysilanes may find novel chiroptical and optoelectronic application.
\end{abstract}

Keywords: Multifunctional polymeric materials; polysilanes; chiral polymers; photoactive polymers.

\section{Introduction}

Now-a-days, technological thrust area addresses the development of multifunctional materials to fulfil the multi-mission objectives by a single material [1-2]. Thus the developed 
multifunctional materials signify to embed multiple functional properties a single polymer material. This could be made possible with designing of materials where the single structures have different functional groups, and/or in the form of organic-inorganic hybrid of several distinct material phases in which each phase performs different necessary function [3]. The synthesis of new multifunctional materials having extraordinary and multifunctional, activity to support the current demand of technological materials is a challenging task.

Polysilanes consist of silicon backbone with organic substituents, which can exhibits extraordinary electronic, optical and photophysical properties such as photoconductivity, UV absorption, solvatochromism, thermochromism, electroluminescence etc. [4-6]. These properties of polysilane have attracted broad attention in academia and industry because of the delocalization of $\sigma$-electron along with the Si-Si chain. The functional materials used in field of semiconductors, precursor for Si-C synthesis, radical photoinitiator and sensors [7]. The direct synthesis of functional polysilanes restricted due to drastic reaction condition (high temperature refluxing of organodichlorosilane monomer along with sodium in toluene) of their synthesis via Wurtz coupling reaction [8-10]. At this refluxing temperature no functional groups sustain with polysilanes. However, functional polysilanes with special functional properties have attracted much more attention to the materials scientists. Hence various routes attempts for the synthesis of functional polysilanes [11]. The post functionalization of polymers is also an alternative way to introduce new functional groups into synthesized polysilanes $[\mathbf{1 1}, \mathbf{1 2 - 1 8}]$. Hydrosilylation reaction verified as an important approach for obtaining functional polysiloxane and the synthesis of functional polysilane [12]. Multihydroxy functional polysilane copolymer synthesized via acetal protecting group strategy [19]. The multifunctional polysilane synthesized by formation of $\mathrm{Si}-\mathrm{Cl}$ intermediate and the substitution of $\mathrm{Cl}$ with hydroxy containing functional NLO chromophore [20]. We have reported functional polysilanes with optically active vinyl monomer, (R)-N-(1phenylethyl) methacrylamide (R-NPEMAM) [21] and functional polysilanes with disperse red 1 methacrylate (DR1MA) [22] synthesized by UU-technique.

In this investigation, multifunctional polysilanes were synthesized by UV irradiation on poly(methylphenylsilane) (PMPS) along with two functional vinyl monomers which are optical active and photoactive monomers. In this synthesis, PMPS gives rise to silyl radicals upon UV-irradiation which initiates the photopolymerization reaction of these functional vinyl monomers such as (R)-N-(1-phenylethyl) methacrylamide (R-NPEMAM) and disperse red 1 methacrylate (DR1MA) respectively. Thus multifunctional a PMPS-block-copolymer (SCDRDM) was consisting of PMPS block along with another block of a random copolymer 
of optically active and photoactive units. The synthesized multifunctional polysilanes were characterized by FTIR and NMR analyses. The molecular weights of the synthesized functional polysilanes were measured by GPC analysis. These synthesized multifunctional polysilanes studied for their optical, chiroptical and photoluminescence properties.

\section{Experimental}

\subsection{Materials}

Dichloromethylphenylsilane (97\%, Sigma Aldrich, USA), sodium metal, THF (Aldrich, USA), n-hexane (Samir Tech Chem. Pvt. Ltd., India), methanol (SDFCL), dichloromethane and acetone (SRL) were used as received. Poly(methylphenylsilane) (PMPS) was synthesized by condensation reaction of dichloromethylphenylsilane with sodium metal dispersion in boiling toluene [23]. (R)-N-(1-phenylethyl)methacrylamide (R-NPEMAM) was synthesized by condensation reaction of methcryloyl chloride and (R)-N-(1-phenylethyl) amine as per the reported procedure [24]. It was further purified by crystallization from ethanol and water (1:1) mixture. The monomer appeared as white crystalline form. Disperse red 1 methacrylate (DR1MA) (95\%, Sigma Aldrich, USA) was purified by crystallization from acetone/ petroleum ether $\left(\mathrm{bp}=60-90^{\circ} \mathrm{C}\right)$ dried and stored at room temperature. Its appearance is red in color [25]. Tetrahydrofuran (THF) purification was mentioned in my published paper [22]. The purified THF was distilled and used immediately.

\subsection{Synthesis of Multifunctional Polysilane containing Optically Active and Photoactive Moieties}

Poly(methylphenylsilane) (PMPS) was used as a macrophotoinitiator which initiated the radical polymerization of optically and photoactive vinyl monomers. In this photopolymerization reaction, PMPS (1.0g), R-NPEMAM (0.189g) and DR1MA (0.382g) were dissolved in $10 \mathrm{ml}$ purified THF in a quartz tube and $\mathrm{N}_{2}$ gas was flushedinto it for 5 minute. Then reactant mixture was sufficiently degassed using a vacuum pump and freeze thaw method. After sealing the tube under reduced pressure,photopolymerization was carried out by exposing it under UV light for 4 minute. (Fusion UV-curing Systems, 10 inch Fusion UV Electrodeless D-bulb, Wavelength $=350-400 \mathrm{~nm}$ ). After UV-exposure, the reaction mixture was kept as such for $24 \mathrm{hrs}$. The reaction mixture was then precipitated in hexane and initially dark orange colour was appeared. Now solvents (hexane and THF) were evaporated. The solid mass was dissolved in acetone and precipitated in methanol. Orange precipitates were observed that was purified by washing through methanol several times 
during the filtration. It is well known that DR1MA and R-N-PEMAM are dissolved in acetone. So this precipitate must be the synthesized functional polymer and block copolymer of PMPS and dispersered1methacrylate and (R)-N-(1-phenylethyl)methacrylamide (SCDRDM). It was dried under vacuum at $50^{\circ} \mathrm{C}$ (Scheme-1). The synthesized sample is SCDRDM-1B. Similarly, other functional polysilanes were synthesized having other composition of reactant ratios: PMPS/DR1MA/R-NPEMAM=1.00g/0.378g/0.764g and $1.00 \mathrm{~g} / 0.668 \mathrm{~g} / 1.026 \mathrm{~g}$ respectively. The synthesized materials are designated as SCDRDM-1B, SCDRDM-2B and SCDRDM-3B. The yield of the synthesized materials was 30-35\%.
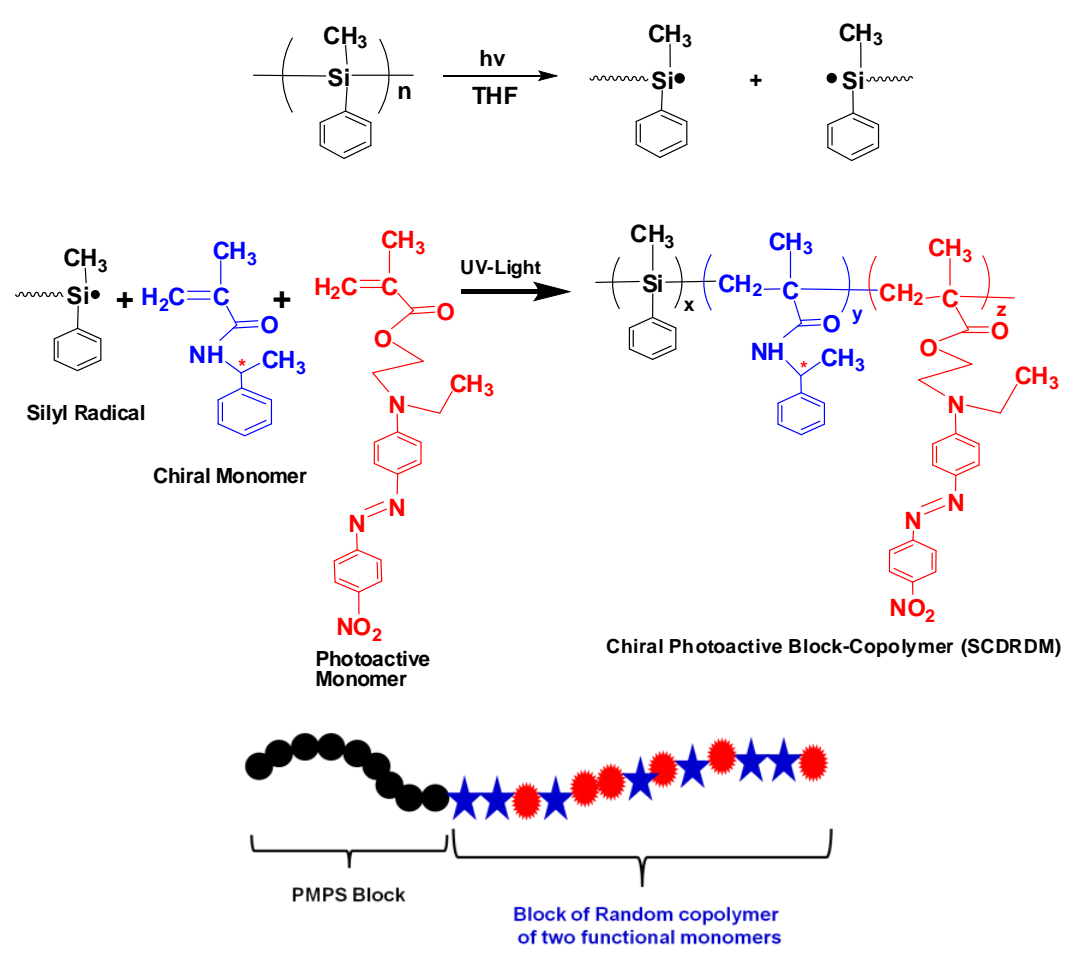

\section{Scheme 1 Synthesis of multifunctional polysilanes having optically active and photoactive moieties}

\subsection{Characterization}

The synthesized multifunctional polysilanes (SCDRDM) were characterized by FTIR and NMR spectroscopy. FTIR spectra of the samples were measured with KBr Pallet on a Perkin Elmer FTIR Spectrum 100. The ${ }^{1}$ H NMR measurements were carried out on a Bruker DRX$300 \mathrm{MHz}$ FT NMR spectrometer in $\mathrm{CDCl}_{3}$ solvent using TMS as the internal reference. The UV-Vis spectra were recorded on an instrument from Analytek Jena Specord 200 PLUS spectrophotometer in THF solution of the samples. The UV-Vis spectra were also taken with variation of temperature from $10^{\circ} \mathrm{C}$ to $60^{\circ} \mathrm{C}$. The molecular weights of the synthesized multifunctional polysilanes (SCDRDM) were measured by using high temperature gel 
permeation chromatography (GPC) (ViscoTek, Malvern, model US make). DSC analysis was carried out using a DSC Q200 (TA Instrument) at a heating rate of $10^{\circ} \mathrm{C} / \mathrm{min}$ under $\mathrm{N}_{2}$ atmosphere. The photoluminescence properties were studied for the synthesized multifunctional polysilanes in their THF solutions with F-2500 FL Spectrophotometer (Hitachi). The circular dichorism (CD) of the synthesized polymer was measured on a JASCO J-810L CD spectropolarimeter.

\section{Results and Discussion}

\subsection{Solubility of Synthesized Multifunctional Polysilanes}

The synthesized multifunctional polysilanes were soluble in THF, chloroform, toluene and DCM but were insoluble in dimethylsulphoxide (DMSO), di-ethylether, acetone, petroleum ether, methanol and hexane.

\subsection{FTIR Analysis}

FTIR spectra of SCDRDM samples are shown in Figure 1. A wide absorption peak at 3444 $\mathrm{cm}^{-1}$ to $3432 \mathrm{~cm}^{-1}$ are the characteristic of NH trans stretching vibration of secondary amide group of chiral mer unit. The narrow band at about $1638 \mathrm{~cm}^{-1}$ is due to $\mathrm{C}=\mathrm{O}$ stretching vibration of - $\mathrm{CONH}$ - of the chiral monomer. On the other hand, the $\mathrm{C}=\mathrm{O}$ of ester group of DR1MA is appeared at $1725 \mathrm{~cm}^{-1}$ in above series. The asymmetric stretching of $\mathrm{NO}_{2}$ group is appeared at about $1481 \mathrm{~cm}^{-1}$, whereas the symmetric stretching of the groupis appeared at $1386 \mathrm{~cm}^{-1}$. The characteristic azo $(-\mathrm{N}=\mathrm{N}-)$ stretching is observed at $1427 \mathrm{~cm}^{-1}$. The other characteristic peak at $1261 \mathrm{~cm}^{-1}$ is appeared due to O-CO of the ester group of DR1MA. The CONHR is appeared at $1527 \mathrm{~cm}^{-1}$, but it is not observed as it is merged with $\mathrm{C}=\mathrm{O}$ (amide) stretching vibration at $1654 \mathrm{~cm}^{-1}$. The Si-Si bond of PMPS block is appeared at $462 \mathrm{~cm}^{-1}$ in all the synthesized samples. The peak appeared at around $1261 \mathrm{~cm}^{-1}$ is due to $\mathrm{Si}-\mathrm{CH}_{3}$ group. The $\mathrm{Si}-\mathrm{CH}_{3}$ wagging vibration is appeared at about $872 \mathrm{~cm}^{-1}$ for all the copolymers. The high intense peak appeared at $731 \mathrm{~cm}^{-1}$ is due to aromatic $\mathrm{C}-\mathrm{H}$ out of plane bending. The peak appeared at $1021-1100 \mathrm{~cm}^{-1}$ is due to $\mathrm{C}-\mathrm{H}$ bending in plane. The $\mathrm{C}-\mathrm{H}$ stretching of methyl group and the alkyl chain $\left(-\mathrm{CH}_{2}-\right)$ all appeared at $2852-2960 \mathrm{~cm}^{-1}$. The $\mathrm{C}-\mathrm{H}$ out of plane bending of aromatic near $\mathrm{NO}_{2}$ resonated around at $876 \mathrm{~cm}^{-1}$. On the other hand, the characterizing stretching frequency of double bond of vinyl $(\mathrm{C}=\mathrm{C})$ around $1614 \mathrm{~cm}^{-1}$ and the absorption band at $928 \mathrm{~cm}^{-1}$ due to the $\mathrm{C}-\mathrm{H}$ bending of vinyl group $\left(=\mathrm{CH}_{2}\right)$ were absent in the spectra. So it clearly indicated the formation of polymer by photoinitiated radical polymerization. 


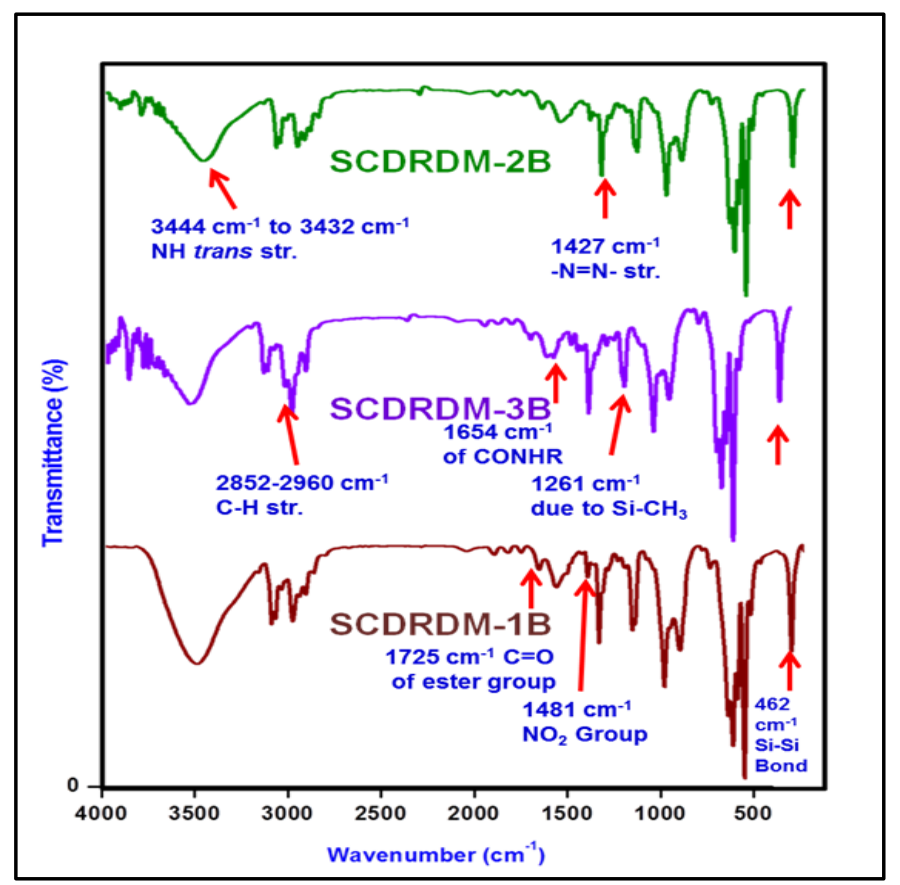

Figure 1 FTIR spectra of multifunctional polysilanes

\subsection{NMR Analysis}

The ${ }^{1} \mathrm{H}$ NMR spectrum of a multifunctional polysilane copolymer (SCDRDM-2B) is shown in Figure 2. The peak appeared at $1.4 \mathrm{ppm}$ as a doublet is due to $\mathrm{CH}_{3}$ of $\mathrm{CH}_{3}-\mathrm{CH}$ group present in chiral unit and also present in photoactive unit. The peak appeared at 0.1 to -0.9 ppm is due to $\mathrm{Si}_{-} \mathrm{CH}_{3}$ group. The signal between 0.77 to $0.92 \mathrm{ppm}$ is exhibited by the proton of $\mathrm{CH}_{3}$ group present in the main polymeric chain. The broad peak observed at 6.5 and 7.5 ppm corresponds to different aromatic protons in the multifunctional polymers present in PMPS, chiral unit (R-NPEMAM) and photoactive unit (DR1MA). The other aromatic peaks appeared at 7.6-7.7 ppm are due to $\mathrm{C}_{6} \mathrm{H}_{5}$ attached with $-\mathrm{N}=\mathrm{N}-$ (azo) group. The peak appeared at 4.23 due to $\mathrm{COO}-\mathrm{CH}_{2}-\mathrm{CH}_{2}$ group of DR1MA unit. The appearance at $0.45 \mathrm{ppm}$ due to $\mathrm{Si}-\mathrm{CH}_{2}$ clearly indicates the formation of block copolymers. The proton of aliphatic $\mathrm{CH}_{2}$ - of polymeric chain appeared in copolymer at $1.68 \mathrm{ppm}$ which indicates the formation of the multifunctional polysilane block copoymers. The disappearance of two peaks at $5.30 \mathrm{ppm}$ and $5.70 \mathrm{ppm}$ of vinyl protons indicates the absence of residual vinyl monomer and formation of functional polymer.

\section{Copolymer Composition of Multifunctional Polysilane}

Copolymer compositions of synthesized multifunctional polysilane (SCDRDM) were determined accurately from ${ }^{1} \mathrm{H}$ NMR Spectrum. The composition of the chiral and 
photoactive polysilane were calculated with consideration of the internal peak area (I) of the Si- $\mathrm{CH}_{3}$ of poly(methylphenylsilane), $\mathrm{CH}-\mathrm{CH}_{3}$ of $\mathrm{Ph}\left(\mathrm{CH}_{3}\right) \mathrm{CH}$ of the chiral monomer and $\mathrm{CH}_{2}$ of $-\mathrm{COOCH}_{2}-\mathrm{CH}_{2}$ of DR1MA unit. The mole fraction $\left(\mathrm{F}_{1}\right)$ of $\mathrm{Si}_{-} \mathrm{CH}_{3}$ for poly(methylphenylsilane) is $0.511,0.436$ and 0.510 , chiral monomer is $0.372,0.327$ and 0.387 and photoactive monomer is $0.116,0.236$ and 0.102 in synthesized block copolymers for SCDRDM-1B, SCDRDM-2B and SCDRDM-3B respectively. The feed ratio of monomer composition is given in synthesis section $\mathbf{2 . 3}$.

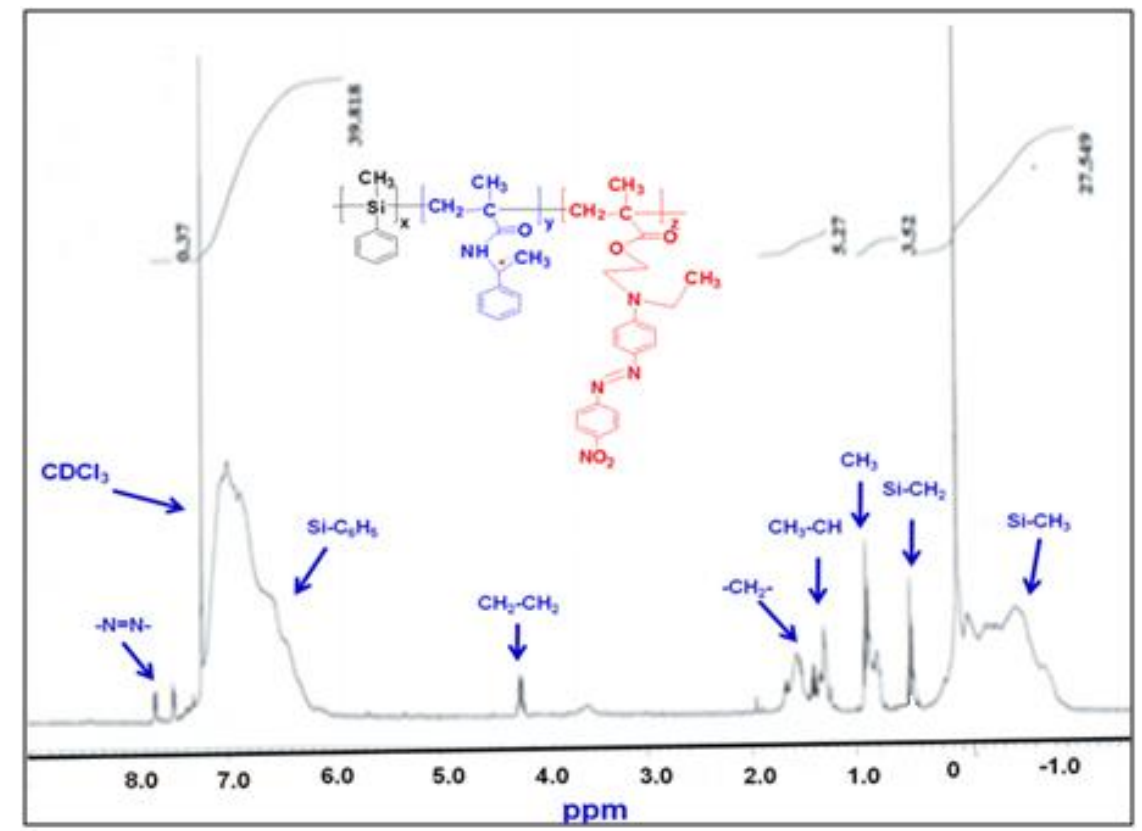

Figure 2 NMR analysis of multifunctional polysilane (SCDRDM-2B)

\subsection{Polymer Molecular Weight}

The molecular weights of PMPS and SCDRDM were measured using gel permeation chromatography (GPC) instrument in THF (Figure 3). In this chromatogram we mentioned the measurement of molecular weight of a UV exposed PMPS sample PSUV-2B for comparison (reported in our published previous paper) [21]. The molecular weight of PMPS is observed to be bimodal distribution which is obvious. Initially, UV exposed sample was observed the unimodal distribution, because of law molecular weight fraction is vanished due to degradation of PMPS chain and formed macroradical on which polymerization of vinyl methacrylate monomers obtained the SCDRDM block copolymer. In second case, the high molecular weight of SCDRDM slightly changed due to uniform Gaussian distribution MWD in comparison to PMPS. 
The number average molecular weight $(\bar{M} \mathrm{n})$ of PMPS and also the functional polymers were measured to be of the order of $10^{3}$ (Table 1). On the other hand, the polydispersity indices of such polymers are 1.99, 1.08, 1.09, 1.09 and 1.10 for PMPS, SCDRDM-1B, SCDRDM-2B and SCDRDM-3B respectively. As a result of UV exposure on PMPS polydispersity indices (PDI) of synthesized SCDRDM is much narrower than PMPS polymer because of chain scission of PMPS, hence molecular weight decreased.

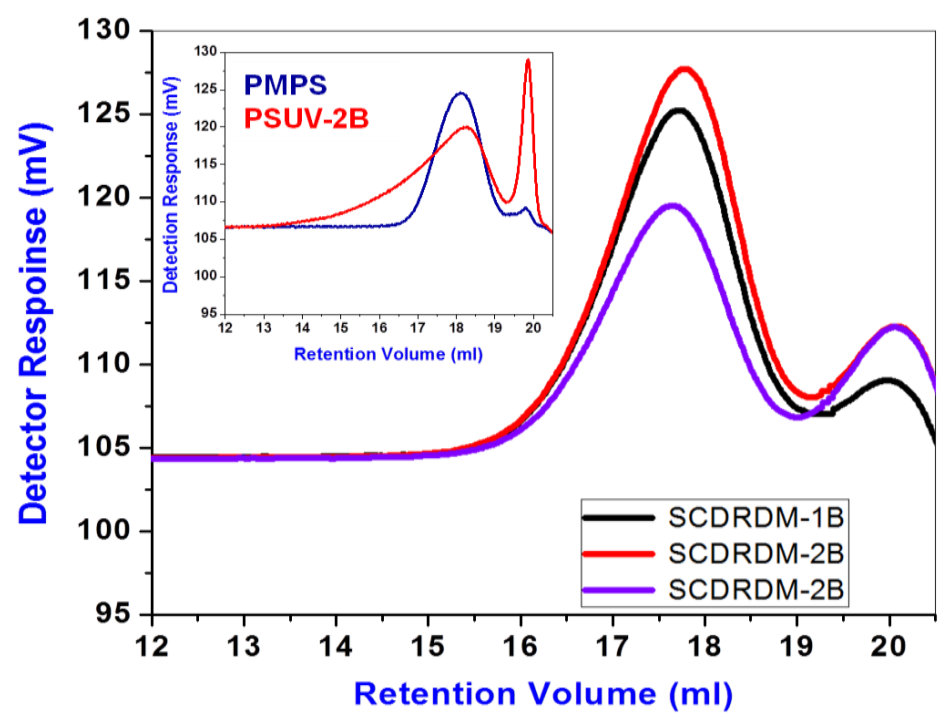

Figure 3 Combined GPC chromatograms of PMPS, UV-exposed PMPS and SCDRDM copolymers

Table1: Molecular Weights of Synthesized Multifunctional PolysilaneCopolymers

\begin{tabular}{|l|l|l|l|l|l|}
\hline Sl. No. & Sample Name & $\bar{M} \mathrm{n}$ & $\bar{M} w$ & $\bar{M} z$ & $\begin{array}{l}\text { PDI }= \\
\bar{M} w / \bar{M} n\end{array}$ \\
\hline 1. & PMPS & 2754 & 5484 & 12049 & 1.991 \\
\hline 2 & SCDRDM-1B & 2202 & 2408 & 2783 & 1.094 \\
\hline 3. & SCDRDM-2B & 2282 & 2498 & 2867 & 1.094 \\
\hline 4. & SCDRDM-3B & 2095 & 2314 & 2690 & 1.104 \\
\hline
\end{tabular}

\subsection{Glass Transition Temperature}

Glass transition temperatures $\left(\mathrm{T}_{\mathrm{g}}\right)$ of the synthesized multifunctional polysilane block copolymers were measured using DSC analysis (Figure 4). The $\mathrm{T}_{\mathrm{g}}$ of homopolymer of DR1MA was reported to be $105.6^{\circ} \mathrm{C}$ [26], and that of chiral polymer (PR-NPEMAM) was reported to be $100.6^{\circ} \mathrm{C}$ [27]. The observed two glass transition temperatures indicated that the synthesized polymers are block copolymers. First block was observed for polysilane block and second block is due to random copolymer of R-NPEMAM and DR1MA functional monomer units. On the other hand, the $\mathrm{T}_{\mathrm{g}}$ of our synthesized PMPS is $62^{\circ} \mathrm{C}$ (Figure 5). The 
synthesized multifunctional polysilane, SCDRDM-1B exhibits two glass transition temperatures at $54.41{ }^{\circ} \mathrm{C}$ and $92.82^{\circ} \mathrm{C}$. First $\mathrm{T}_{\mathrm{g}}$ is associated with the polysilane block and second for the random block of photoactive DR1MA unit and chiral unit. Two glass transition temperatures for SCDRDM-3B sample are observed to be $60.47^{\circ} \mathrm{C}$ and $98.03^{\circ} \mathrm{C}$. The higher value of this block copolymer with respect to first one (SCDRDM-1B) may be due to higher molecular weights. The reported glass transition temperatures of the chiral homopolymer and photoactive homopolymer are more than the random copolymer blocks in this synthesized multifunctional polysilanes. This may be due to different method of synthesis: In the first cases it was radical polymerization and the second case is the UV initiated radical polymerization by silyl macroradical where low $\bar{M} w$ block was formed.

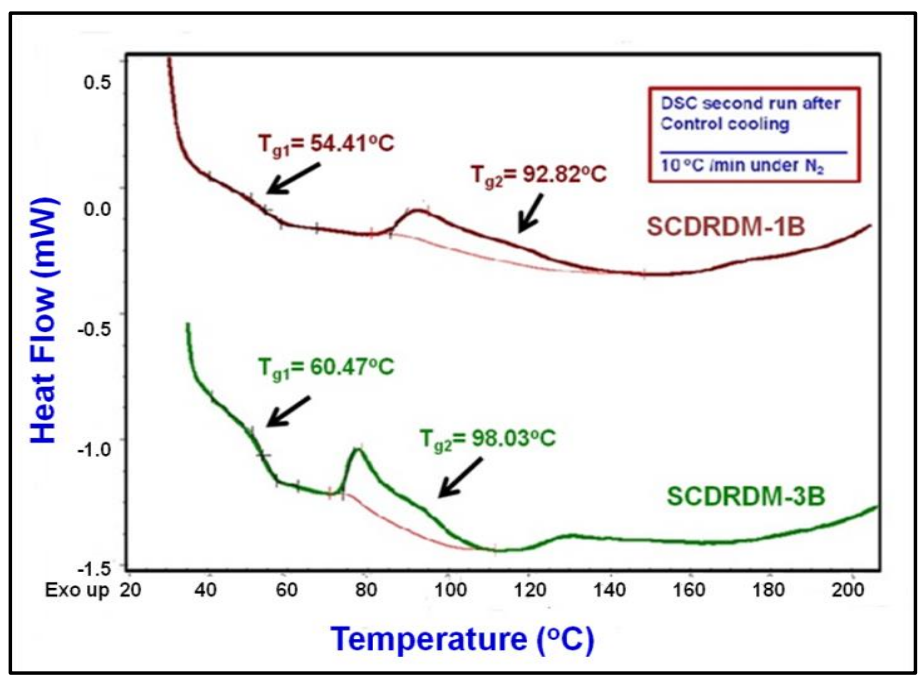

Figure 4 DSC Thermograms of functional polysilane block copolymers

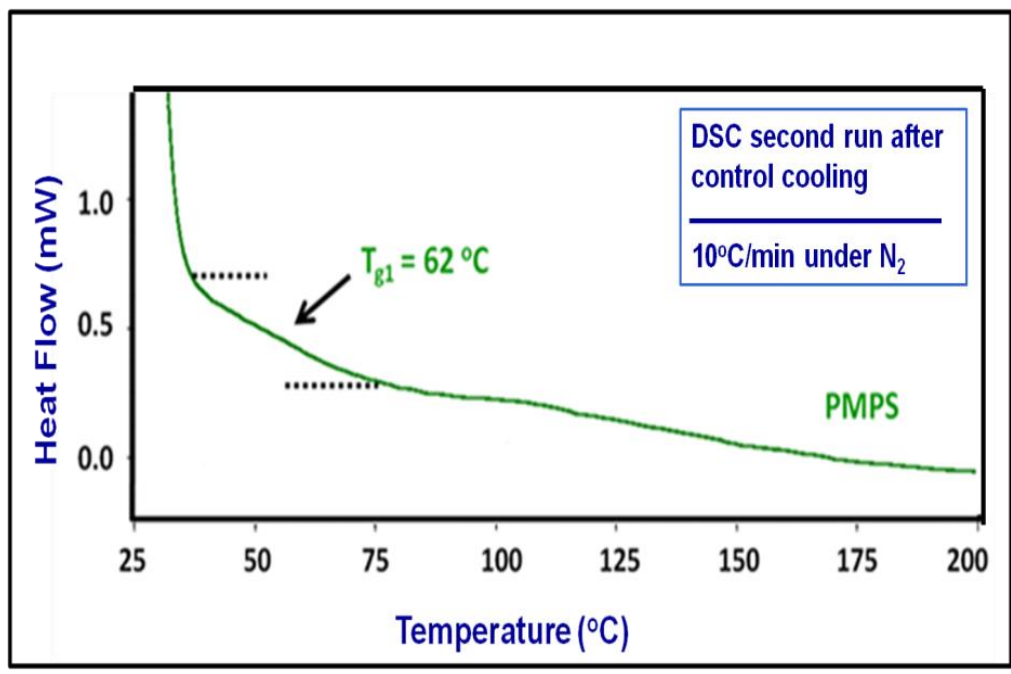

Figure 5 DSC Thermograms of poly(methylphenylsilane) (PMPS) 


\subsection{Optical properties}

The optical absorbance spectra of functional polysilane copolymers (SCDRDM) are shown in Figure 6. The electronic absorption maxima which appeared at $330 \mathrm{~nm}$ is due to $\sigma-\sigma^{*}$ transition of the $\sigma$-conjugation of Si-Si main chain. The absorbance at $272 \mathrm{~nm}$ can be assigned to the $\pi-\pi^{*}$ transition of aromatic ring. The electronic absorption in the visible region at $475 \mathrm{~nm}$ corresponds to the combined contribution of $n-\pi^{*}$, first $\pi-\pi^{*}$ intermolecular charge transfer electronic transition of the azobenzene chromophores of DR1MA unit. The absorbance of this azobenzene chromophore is in corroborated with the reported results $[\mathbf{2 5}$, 28]. The Si-Si backbone of polysilane is sensitive towards external stimuli especially for temperature. The effect of temperature on such properties of the synthesized functional polysilanes was also studied. The temperature effect on the absorbance at $266 \mathrm{~nm}$ due to aromatic ring, at $475 \mathrm{~nm}$ due to DR1MA unit and on the absorbance at $330 \mathrm{~nm}$ due to Si-Si $\sigma$-conjugation is shown in Figure 7 to 9. The absorbance of flexible Si-Si bond which appears at about $330 \mathrm{~nm}$ was decreased marginally with increase in temperature $\left(10^{\circ} \mathrm{C}-60^{\circ} \mathrm{C}\right)$.

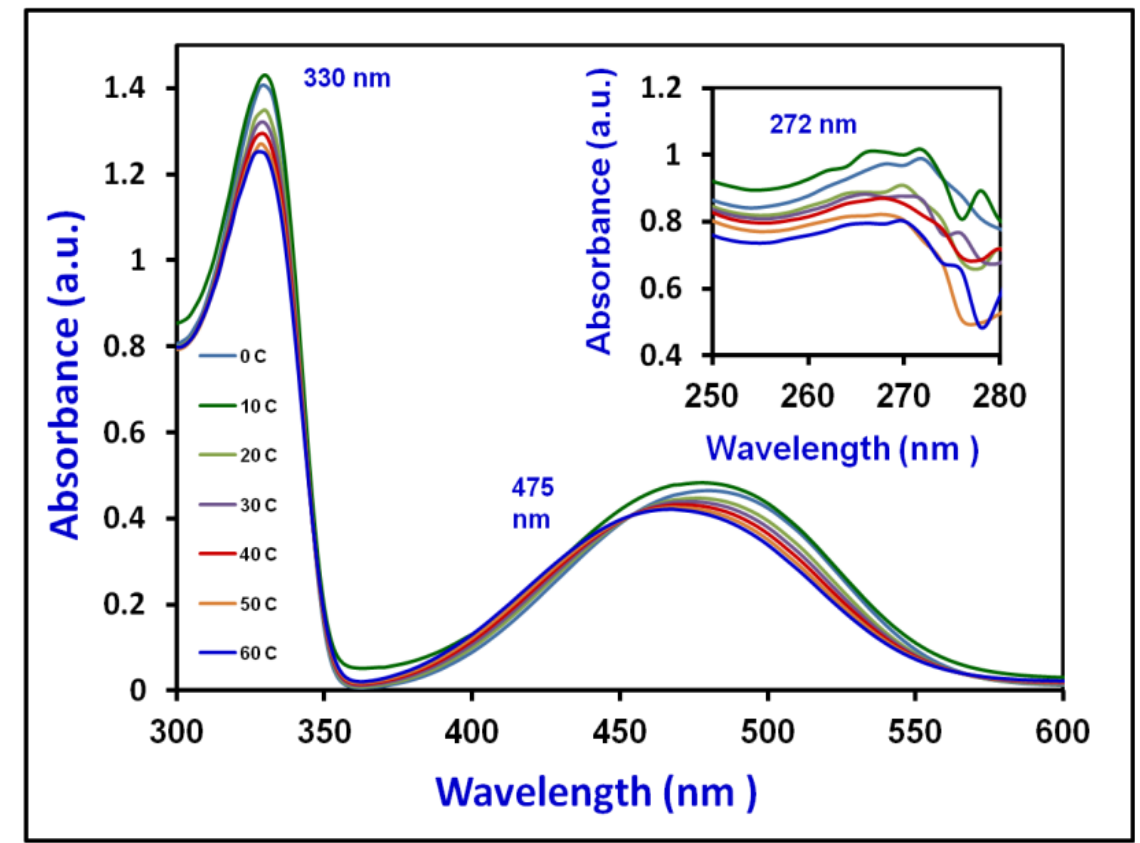

Figure $6 \mathrm{UV}-$ Vis spectra of a functional polysilane (SCDRDM-3B) in THF (conc. $0.125 \mathrm{mg} / \mathrm{dl}$ ) 


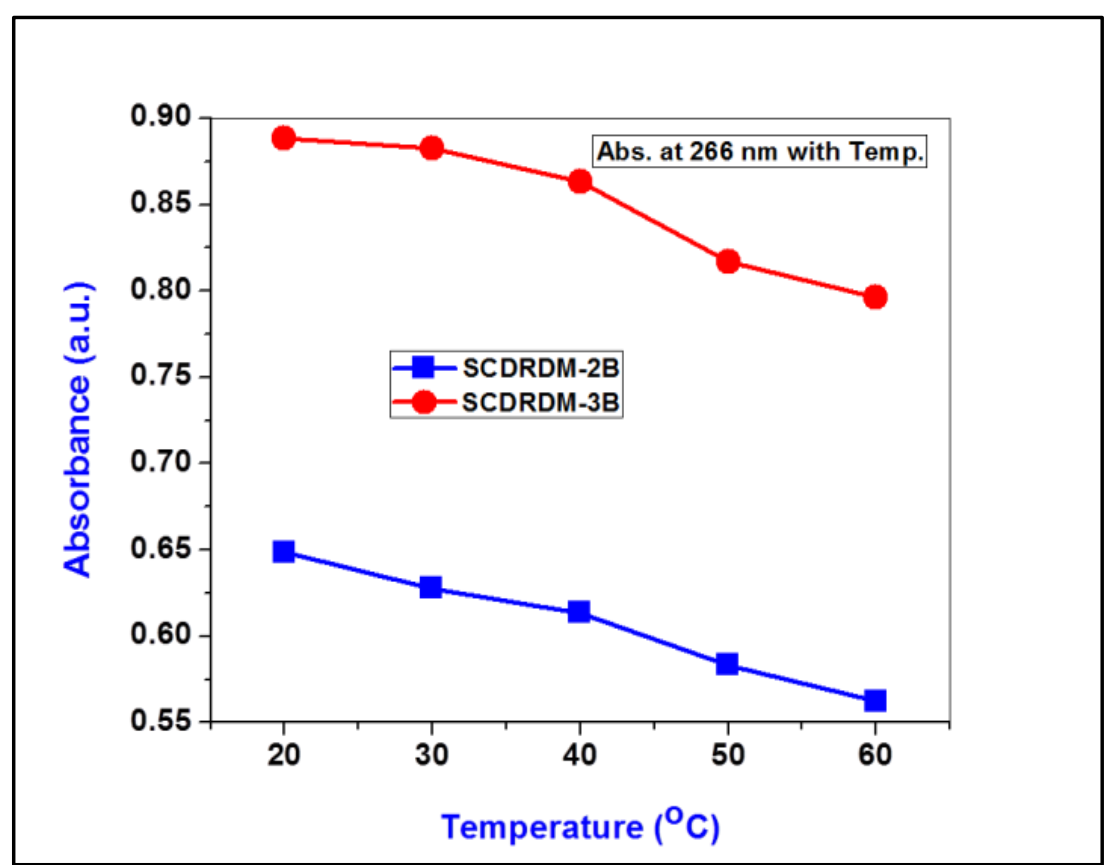

Figure 7 Temperature versus optical absorbance (at $266 \mathrm{~nm}$ due to benzene ring)

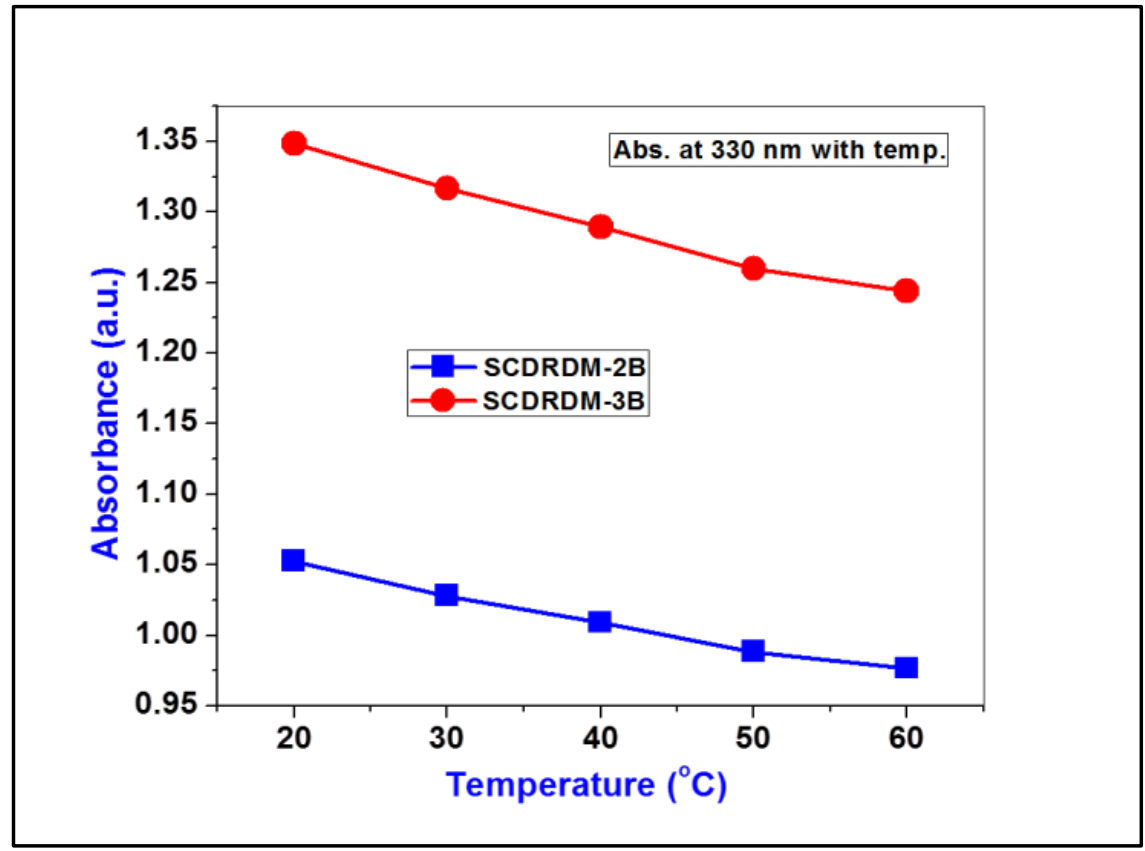

Figure 8 Temperature versus optical absorption (at $330 \mathrm{~nm}$ due to $\mathrm{Si}-\mathrm{Si}$ bond) 


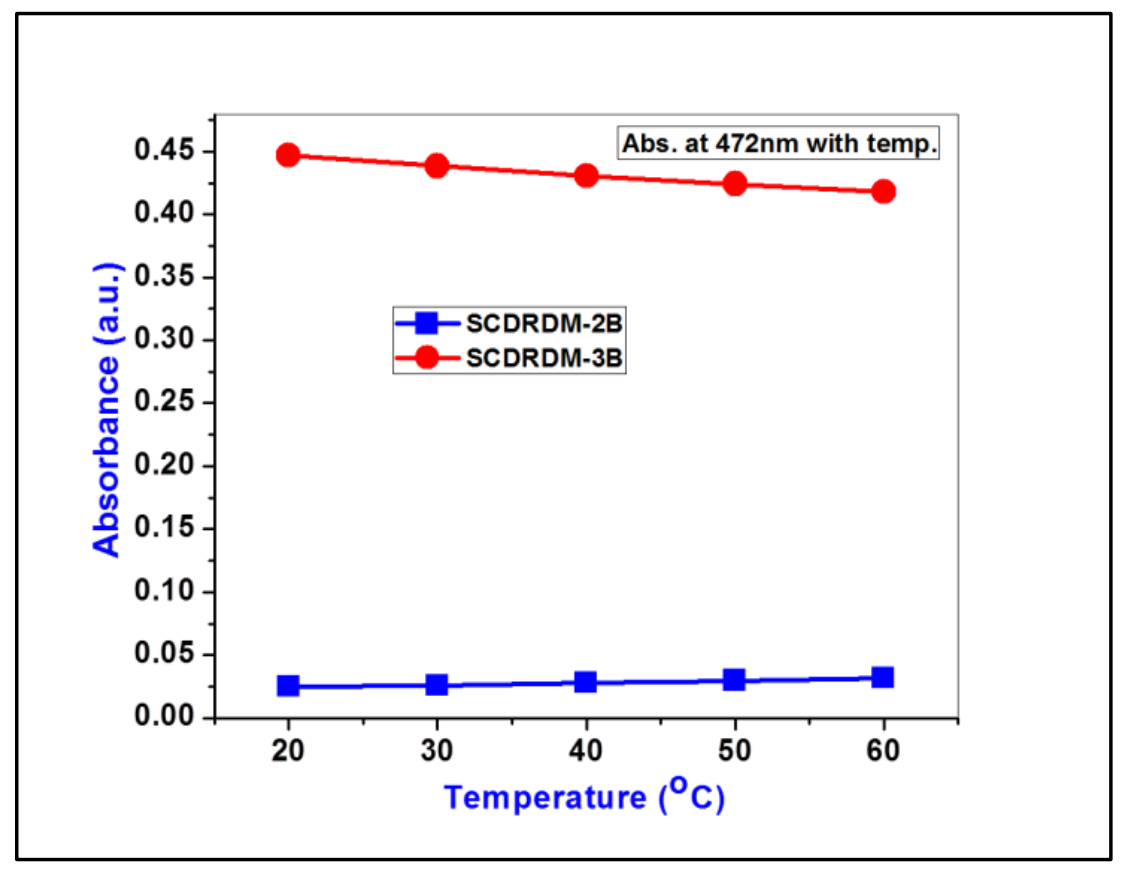

Figure 9 Temperature versus optical absorption (at $426 \mathrm{~nm}$ due to azobenzene group)

\subsection{Chiroptical Properties}

\section{Circular Dichorism (CD)}

The chiroptical properties of functional optically active and photoactive polysilanes were determined using circular dichorism in THF solution in the spectral range of 200 to $700 \mathrm{~nm}$ (Figure 10 and 11). The cotton effect observed at $261 \mathrm{~nm}$ is due to aromatic ring associated with the chiral unit, (R)-N-(1-phenylethyl)methacrylamide and it is obvious [24]. Moreover, we also observed the cotton effect at $330 \mathrm{~nm}$ and $470 \mathrm{~nm}$, which are associated with the Si-Si conjugation of PMPS block and azobenzene chromophore of photoactive monomer unit. Hence these induced circular dichroism signals appeared in case of functional polysilane block copolymers may be due to the presence of chain helicity into $\mathrm{Si}$-Si chain and also other interaction with the azobenzene chromophores of the photoactive DR1MA unit. Thus the synthesized multifunctional polysilanes have tunable chiroptical properties. Polysilanes have one handed helical-screw conformation with chiral alkyl substituents [29-32].

Chain helicity induced in oligosilane by the terminal substituents of chiral unit was also reported [33-34]. Thus polysilane can take a stable conformation in one handed helix sense as a result of chiral stimuli [35-37]. Polysilane- $b$-poly-(triphenylmethyl methacrylate) was also reported to exhibit helical conformation of polysilane block due to helical induction of poly(triphenylmethyl methacrylate) block [38]. It was reported to have cotton effect at 340 
$\mathrm{nm}$ below $-20^{\circ} \mathrm{C}$. The helical sense of one polymer chain could induce helical sense in another polymer chain throughout block copolymer system in response to an external stimuli such solvent, $\mathrm{pH}$ and temperature. It was also reported that polysilane exhibited optical activity with (+) or (-)-menthoxide group at the terminal position due to adoptation a preferential helical sense [39]. The chiral solvent influenced the behaviour of polymerization and polymers which affect the molecular distribution of a helical polymer [40-41]. The polymer chains of poly(methylphenylsilane) and poly(hexylmethylsilane) were adopted preferred helical screw senses through such chiral salvation [41]. In our earlier investigation we observed the circular dichroism due to induction of chain helicity in poly(methylphenylsilane) chain as a result of the presence of chiral block at one or both the ends of chiral-block polysilanes [21]. In this investigation also we observed the induced optical activity both in Si-Si chain of PMPS and in azobenzene chromophore of photoactive monomer (DR1MA) due to the presence of chiral unit in the synthesized multifunctional polysilanes (SCDRDM).

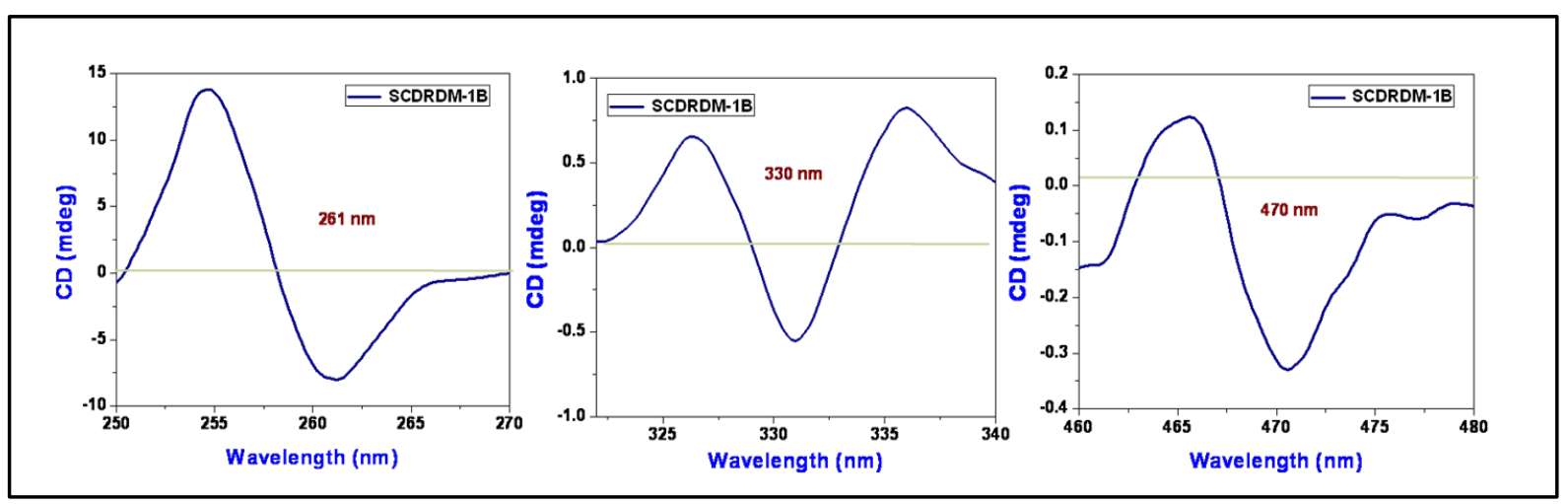

Fig. 10 CD spectra of SCDRDM-1B in THF (conc. 0.0005 gm/dl)

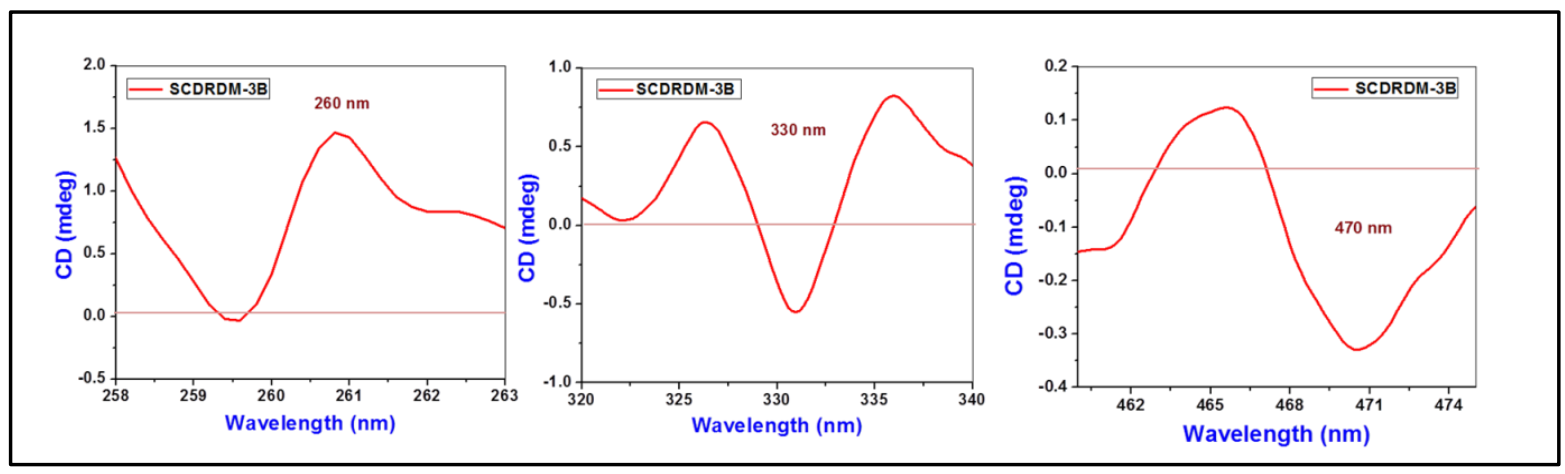

Fig.11 CD spectra of SCDRDM-3B in THF (conc. 0.0005 gm/ml) 


\subsection{Photoluminescence Properties}

The photoluminescence (PL) properties of the synthesized multifunctional polysilane were observed in THF with excitation energy such as $275 \mathrm{~nm}$ due to aromatic ring, $325 \mathrm{~nm}$ for SiSi chain and also $475 \mathrm{~nm}$ for azobenzene chromophore (Figures 12, 13 and 14). The optimum $\mathrm{PL}$ is observed at $\lambda_{\mathrm{em}}=307 \mathrm{~nm}$ for the excitation energy of $275 \mathrm{~nm}$. Another photoemission is observed at $\lambda_{\mathrm{em}}=415 \mathrm{~nm}$ for the same excitation energy. The same emission is also observed at $415 \mathrm{~nm}$ for the excitation at $325 \mathrm{~nm}$. The PL intensity is enormously increased due to decrease in the solution concentration. But there is no shift of emission wavelengths due to change in concentration of the solution.

Other than the obvious emissions as mentioned above, a multiple emission spectra are also observed at $500 \mathrm{~nm}, 550 \mathrm{~nm}$ and $590 \mathrm{~nm}$ due to excitation at $475 \mathrm{~nm}$. This may be due to the exciton coupling between the azobenzene chromophore of DR1MA and benzene ring of RNPEMAM block with Si-Si $\sigma$-conjugation in association with the aromatic ring of PMPS block (Scheme 2). Such synthesized functional polysilanes having unusual photoluminescence property may find novel optoelectronics application. The appearance of fluorescent band was reported at around $360 \mathrm{~nm}$, which is assigned to a fluorescence from their lowest excited state $\sigma-\sigma^{*}$ transition of Si-Si chain [42]. This sharp photoluminescence $\mathrm{UV}$ band is of excitonic nature, which is associated along with the Si-Si backbone [43]. A broad visible photoluminescence (BVPL) band is also reported at $380 \mathrm{~nm}$ regions and its maximum is situated at about $415 \mathrm{~nm}$ [43-44]. The contribution of PMPS block of the copolymers and both exciton photoluminescence (EPL) and broad visible photoluminescence (BVPL) band are observed at around $350 \mathrm{~nm}$ and $415 \mathrm{~nm}$ in functional polysilane block copolymers (SCDRDM) when excited at $325 \mathrm{~nm}$ (Figure 13). The photoluminescence of DR1MA monomer and its polymer was reported in which photoemission was observed at around $320 \mathrm{~nm}$ [26]. Photoluminescence behavior of copolymers of DR1MA with a chiral monomer was reported that exhibited optimum photoluminescence at around $307 \mathrm{~nm}$ due to excitation from $275 \mathrm{~nm}$. A blue shift of 10-12 nm was observed for the copolymers of DR1MA [25]. It is true that the $\pi-\pi$ stacking arising for two azobenzene moieties was minimized due to second monomer introduction (chiral monomer) in the copolymers. This blueshift may not have only due to $\pi-\pi$ stacking of a similar kind of moiety, such as, azobenzenes, but also to the randomly distributed $\pi-\pi$ stacking of the benzene ring of the chiral comonomer and azobenzene chromophore of DR1MA [25]. In this investigation when excited from $275 \mathrm{~nm}$, we also observed emission at $307 \mathrm{~nm}$ due to DR1MA block along with PMPS block at around $415 \mathrm{~nm}$ (Figure 12). Other than the obvious multiple emissions spectra 
are also observed centred at $550 \mathrm{~nm}$ due to excitation at $475 \mathrm{~nm}$ (Figure 14). This may be due to the exciton coupling between the azobenzene chromophore of DR1MA block and Si-Si $\sigma$ conjugation in association with the aromatic ring of PMPS block (Scheme 2). Such synthesized functional photoactive polysilane block copolymers having unusual photoluminescence property may find novel optoelectronics application.

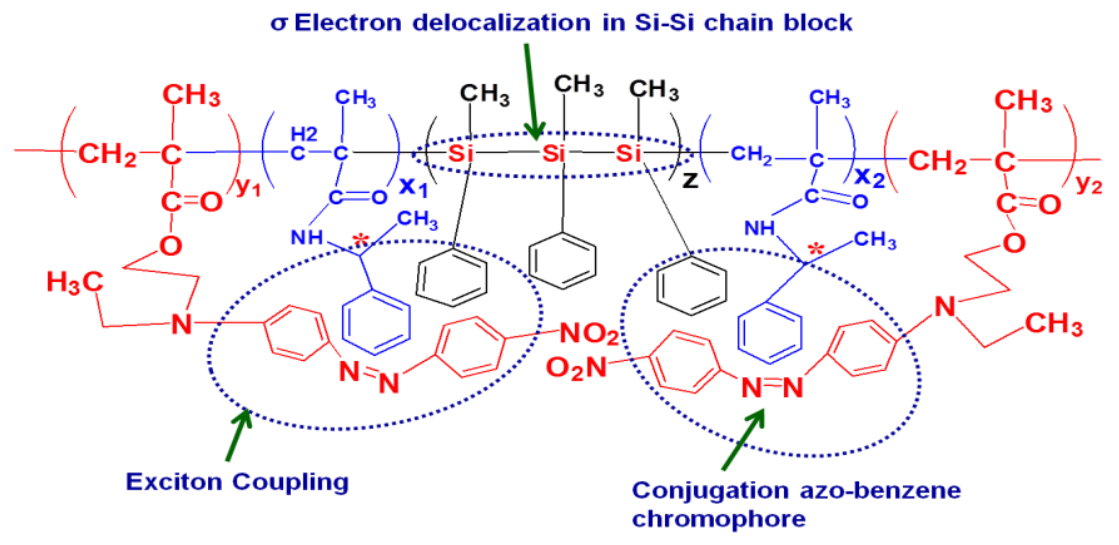

Scheme 2 Plausible interaction of functional moieties in synthesized functional polysilanes (SCDRDM)

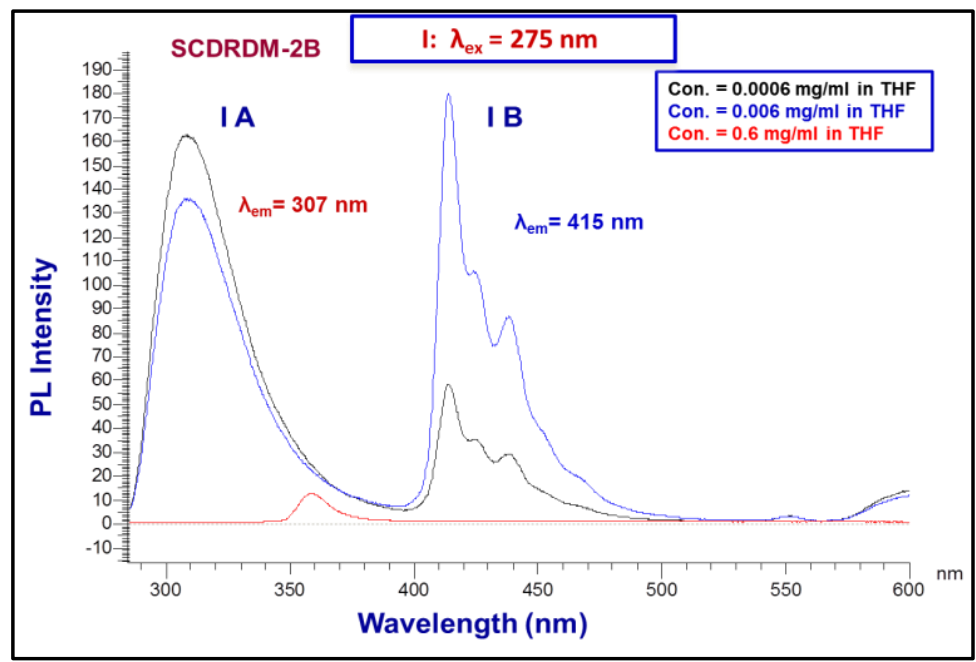

Figure 12 PL spectra of SCDRDM-2B with excitation energy of $275 \mathrm{~nm}$ 


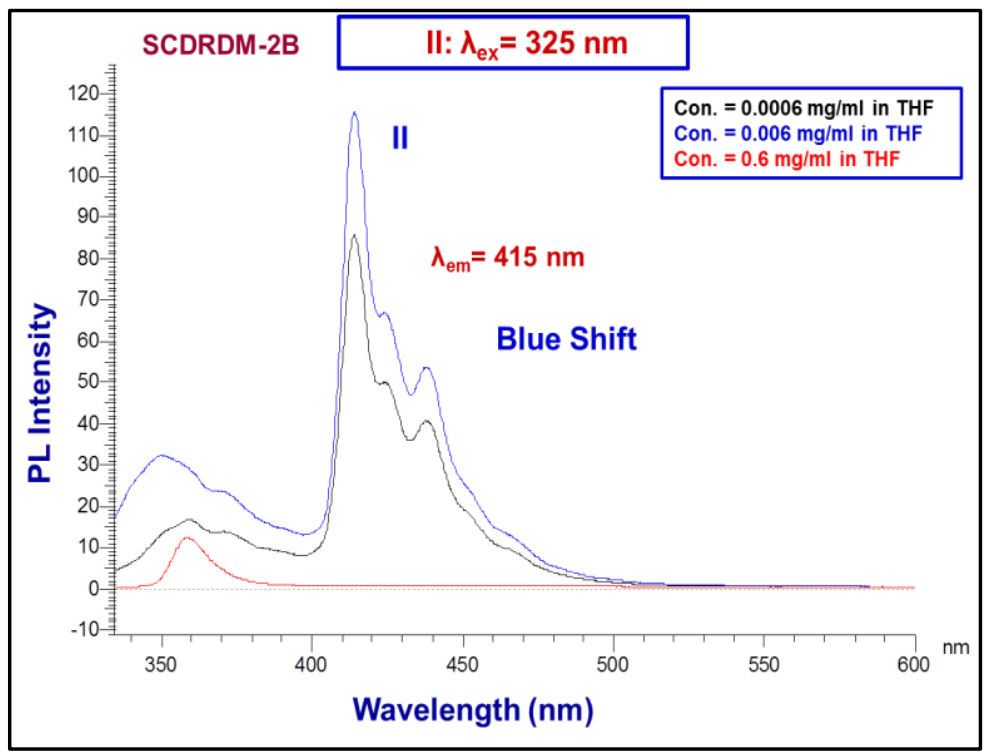

Figure 13 PL spectra of SCDRDM-2B with excitation energy of $325 \mathrm{~nm}$

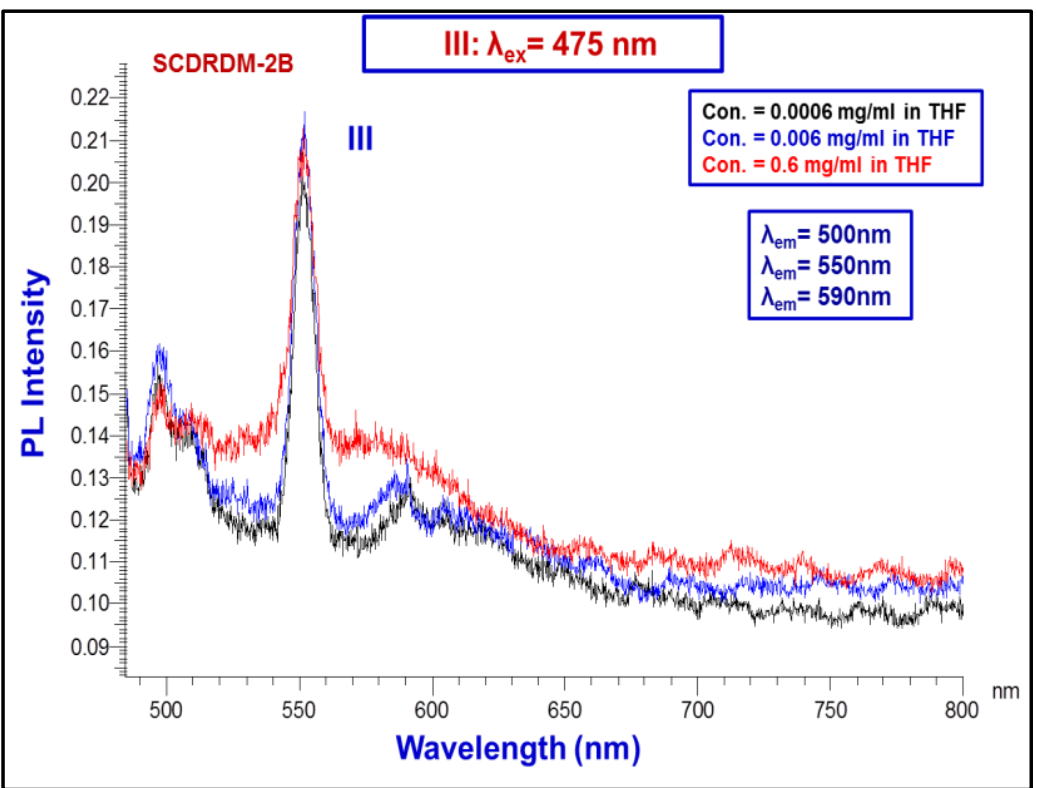

Figure 14 PL spectra of SCDRDM-2B with excitation energy of $475 \mathrm{~nm}$

Table 2 Results of PL Analysis of Photoactive Polysilane Block Copolymer (SCDRDM-2B)

\begin{tabular}{|c|c|c|c|c|}
\hline \multirow{2}{*}{$\begin{array}{l}\text { Conc. } \\
\text { Polymer } \\
\text { Solution } \\
(\mathrm{mg} / \mathrm{ml})\end{array}$} & \multicolumn{2}{|c|}{ Excitation $\left(\lambda_{\mathrm{ex}}=275 \mathrm{~nm}\right)$} & \multicolumn{2}{|c|}{ Excitation $\left(\lambda_{\mathrm{ex}}=325 \mathrm{~nm}\right)$} \\
\hline & $\begin{array}{l}\lambda_{\mathrm{em}} \\
(\mathrm{nm})\end{array}$ & $\begin{array}{l}\text { PL intensity } \\
\text { (au) }\end{array}$ & $\begin{array}{l}\lambda_{\mathrm{em}} \\
(\mathrm{nm})\end{array}$ & $\begin{array}{l}\text { PL intensity } \\
(\mathrm{au})\end{array}$ \\
\hline $6 \times 10^{-4}$ & 307 & 163 & 352 & 32 \\
\hline $6 \times 10^{-3}$ & 307 & 136 & 359 & 17 \\
\hline $6 \times 10^{-4}$ & 415 & 180 & 415 & 116 \\
\hline $6 \times 10^{-3}$ & 415 & 57 & 415 & 86 \\
\hline
\end{tabular}




\section{Conclusion}

Multifunctional polysilanes were synthesized along with a chiral monomer, R-NPEMAM and a photoactive monomer, DR1MA. By the UV irradiation, PMPS gave rise to silyl radical, which initiated by radical polymerization with (R)-N-(1-phenylethyl)methacrylamide (R-NPEMAM) and disperse red 1 methacrylate (DR1MA) monomers. The number average molecular weights $(\bar{M} \mathrm{n})$ of PMPS and SCDRDM-1B, SCDRDM-2B, SCDRDM-3B were measured to be $2.75 \times 10^{3}, 2.20 \times 10^{3}$, $2.28 \times 10^{3}$ and $2.09 \times 10^{3}$ respectively. In the UV-Vis spectra, peak appeared at $272 \mathrm{~nm}$ is due to presence of aromatic ring. The absorption due to $\mathrm{Si}-\mathrm{Si} \sigma$-conjugation appeared at $330 \mathrm{~nm}$. The electronic absorption in the visible region at $475 \mathrm{~nm}$ corresponds to the combined contribution of $\mathrm{n}$ $\pi^{*}$, first $\pi-\pi^{*}$ intermolecular charge transfer electronic transition of the azobenzene chromophores of DR1MA unit. These synthesized multifunctional polymers not only exhibited circular dichroism due to the chiral unit (at $261 \mathrm{~nm}$ ) present in the polymeric chain, but also exhibited induced circular dichroism at $330 \mathrm{~nm}$ and $470 \mathrm{~nm}$ associated with the Si-Si chain of PMPS and azobenzene chromophore of DR1MA unit, respectively. This type of tuneable optical activity may find novel application. These synthesized functional polysilanes exhibited good photoluminescence properties. They emitted $307 \mathrm{~nm}$ when excited at $275 \mathrm{~nm}$. Another photoemission is observed at $\lambda_{\mathrm{em}}=415 \mathrm{~nm}$ for the same excitation energy. The same emission at $415 \mathrm{~nm}$ is also observed for the excitation at $325 \mathrm{~nm}$. Other than, the obvious emissions, multiple emission spectra are also observed at $500 \mathrm{~nm}$, $550 \mathrm{~nm}$ and $590 \mathrm{~nm}$ for the excitation at $475 \mathrm{~nm}$. This might be due to exciton coupling between azobenzene chromophore, benzene ring and $\mathrm{Si}-\mathrm{Si}$ conjugation present in the multifunction polysilanes. Such synthesized multifunctional polysilanes having unusual optical activity as well as photoluminescence property may find novel optoelectronic applications.

\section{Acknowledgements}

The authors gratefully acknowledge the encouragement and support of Dr. N. Eswara Prasad Director, DMSRDE, to pursue this task. Dr. D. N. Tripathi Sc-'G' and Dr. T.C. Shami Sc-'F' is also acknowledged for his valuable suggestion and support.

\section{References:}

1. S. Nemat-Nasser, T. Plaisted, A. Starr and A. V. Amirkhizi “Multifunctional Materials" in BIOMIMETICS: Biologically Inspired Technologies,” Yoseph Bar-Cohen (Ed.), CRC Press 2005, Ch.12, pp.309-341

2. D. S. Bag, “Functional Polymers" in "Principles of Polymers-An advanced Book," D. S. Bag, Nova Science Publishers Inc., New York, 2013, Ch.11, pp.497-590 
3. J. Li, Z. Li, H. Tang, H. Zeng, J. Qin, "Polysilanes with NLO Chromophores as Pendant Groups by Utilizing Different Synthetic Strategies,” J. Organomet. Chem. 685 (2003) 258268

4. R.D. miller, J. Michl, “Polysilane High Polymers,” Chem. Rev. 89 (1989) 1359-1410

5. R. West, “The Polysilane High Polymers,” J. Organomet. Chem. 300 (1986) 327-346

6. K. Matyjaszewski, M. Cypryk, H. Frey, J. Hrkach, H.K. Kim, M. Moller, K. Ruel, M. White, “Synthesis and Characterization of Polysilanes,” J. Macromol. Sci.A: Pure Appl. Chem. 28 (1991) 1151-1176

7. R.West, R. Menescal, T. Asuke, J. Eveland, "Some recent developments in polysilane chemistry,” J. Inorg. Organomet. Polym. 2 (1992) 29-45

8. S.H. Yi, N. Maeda, T. Suzuki, H. Sato, "Preparation and Characterization of Polysilanes with Electron Donating Substituent,” Polym. J. 24 (1992) 865-870

9. T.J. Cleij, J.K. King, L.W. Jenneskens, “Band Gap Modifications in Functionalized Poly(methylphenylsilanes),” Macromolecules, 33 (2000) 89-96

10. L.A. Harrah, J.M. Zeigler, “Electronic Spectra of Hindered Silyl and Organo-substituted Polysilylenes,” Macromolecules, 20 (1987) 2037-2039

11. J. Koe, “Contemporary Polysilane Synthesis and Functionalisation,” Polym. Int. 58 (2009) 255-260

12. L.A. Schwegler, C. Meyer-Pundsack, M. Moller, "Synthesis of Polyethylene Oxide Graft Polysilylenes and Networks Based on Poly(Di-N-Pentylsilylene/N-Pentyloct-7-Enyl) Copolymers,” J. Polym. Sci. Part A: Polym. Chem. 38 (2000) 2306-2318

13. H. Ban, K. Sukegawa, S. Tagawa, "Pulse Radiolysis Study on Organopolysilane Radical Anions,” Macromolecules, 20 (1987) 1775-1778

14. E. Hansdrickx, D.V. Steenwinckel, A. Persoons, “Photorefractive Polysilanes Functionalized with a Nonlinear Optical Chromophore,” Macromolecules, 32 (1999) 22322238

15. C. Zhan, H. Zeng, J.Qin, D. Liu, N. Cheng, Y. Cui, "Synthesis, Photoconduction and Electro-Optical Effect of a Polysilane with Disperse Red-1 As Side Chain,” Synth. Met. 84 (1997) 397-398

16. H. Tang, Y. Liu, B. Huang, J. Qin, C. F.Hernandez, B. Kippelen, S. Lid and C. Ye, "Synthesis and Optical Properties of a Series of Chromophore Functionalized Polysilanes," J. Mater. Chem., 15 (2005) 778-784 
17. Z.H. Peng, Z.N. Bao, Y.M. Chen, L. Yu, Large “Photorefractivity in an Exceptionally Thermostable Multifunctional Polyimide,” J. Am. Chem. Soc. 116 (1994) 6003-6004

18. Z.H. Peng, A.R. Gharavi, L.Yu, “Hybridized Approach to New Polymers Exhibiting Large Photorefractivity,”Appl. Phys. Lett. 69 (1996) 4002-4004

19. V. S. Reuss and H. Frey, "Multihydroxy-Functional Polysilanes via an Acetal Protecting Group Strategy,” Macromolecules, 43 (2010) 8462-8467

20. H.Tang, J. Li, J. Qin, "Synthesis of Multifunctional Polysilanes via Si-Cl Containing Intermediate," Reactive and Funct. Polym. 48 (2001) 193-199

21. Km. Meenu, D.S. Bag, A. K. Saxena, "Synthesis of Organic-Inorganic Chiral block Poly(Methylphenylsilane) Functional Polymers, and Study of Their Optical and Chiroptical Properties,” J. of Polym. Sci. Part A: Polym. Chem. 54 (2016) 3626-3634

22. Km. Meenu, Bag, D. S., "Synthesis and Characterization of Functional Photoactive Organic-Inorganic Block Copolymers of Poly(Methylphenylsilane) and Disperse Red 1 Methacrylate and Study of Their Optical and Photophysical Properties,” J. Macromol. Sci.: Pure and Appl. Chem. 54 (2017) 418-425

23. K. Matsukawa, S. Fukui, N. Higashi, M. Niwa, H. Inoue, “Preparation and Properties of Organic-Inorganic Hybrid Thin Films Containing Polysilane Segments from Polysilanemethacrylate Copolymers,” Chem. Lett.28 (1999) 1073-1074

24. D.S. Bag, T.C. Shami and K.U. Bhasker Rao, "Synthesis and characterization of a chiral monomer and its polymer, poly [D-(+)-a-methyl benzyl methacryloylamine],” J. Polym. Mater. 25 (2008) 51-62

25. D.S. Bag and S. Alam, "Synthesis and Characterization of Photoactive Chiral Copolymers of (S)-N-(1-Phenyl Ethyl) Methacrylamide and Disperse Red 1 Methacrylate,” J. Appl. Polym. Sci.125 (2012) 2595-2603

26. L. Ding, T.P. Russell, “A Photoactive Polymer with Azobenzene Chromophore in the Side Chain,” Macromolecules, 40 (2007) 2267-2270

27. M.M. Green, M.P. Reidy, R. J. Johnson, G. Darling, D.T. O’Leary and G. Wilson, "Macromolecular Stereochemistry: The Out-of-Proportion Influence of Optically Active Comonomers on the Conformational Characteristics of Polyisocyanates. The Sergeants and Soldiers Experiment,” J. Am. Chem. Soc. 111 (1989) 6452-6454

28. A. Altomare, F. Ciardelli, M.S. Ghiloni, R. Solaro, N. Tirelli, “Chiral Methacrylic Polymers Containing Permanent Dipole Azobenzene Chromophores. ${ }^{13} \mathrm{C}$ NMR Spectra and Photochromic Properties,” Macromol. Chem. Phys. 198 (1997) 1739-1752

29. M. Muller and R. Zentel, “Azo-dyes as Labels and as Photoisomerizable Units in Chiral Polyisocyanates,” Macromol. Chem. and Phys. 194 (1993) 101-116 
30. M. Fujiki, “Helix Magic. Thermo-Driven Chiroptical Switching and Screw-Sense Inversion of Flexible Rod Helical Polysilylenes,” J. Am. Chem. Soc. 122 (2000) 3336-3343

31. J. R. Koe, M. Fujiki and H. Nakashima, "First Optically Active Diarylpolysilanes: Facile Helical Screw Sense Control with Only (S)-Enantiopure Side Chains,” J. Am. Chem. Soc. 121 (1999) 9734-9735

32. H. Frey, M. Mollerand K. Matyjaszewski, “Chiral Poly(dipentylsilylene) Copolymers,” Macromolecules, 27 (1994) 1814-1818

33. K. Obata and M. Kira, "Temperature Dependent CD Spectra of Poly(dihexylsilylene)s with Terminal Chiral Groups. Coupled Cotton Effects of Silicon $\sigma$ Chains,” Macromolecules, 31(1998) 4666-4668

34. K. Obata and M. Kira, "Induction of Helical Chirality in Linear Oligosilanes by Terminal Chiral Substituents,” J. Am. Chem. Soc. 119 (1997) 11345-11346.

35. J. R. Koe, M. Fujiki, M. Motonaga, and H. Nakashima, “Temperature-Dependent HelixHelix Transition of an Optically Active Poly(Diarylsilylene)," Chem.Commun. (2000) 389390

36. H. Nakashima, J. R. Koe, K. Torimitsu, and M. Fujiki, “Transfer and Amplification of Chiral Molecular Information to Polysilylene Aggregates,” J. Am. Chem. Soc. 123 (2001) 4847-4848

37. H. Nakashima, M. Fujiki, J. R. Koe and M. Motonaga, "Solvent and Temperature Effects on the Chiral Aggregation of Poly(alkylarylsilane)s Bearing Remote Chiral Groups,” J. Am. Chem. Soc. 123 (2001) 1963-1969.

38. T. Sanji, K. Takase and H. Sakurai, “Helical-Sense Programming through PolysilanePoly(triphenylmethyl methacrylate) Block Copolymers,” J. Am. Chem. Soc. 123 (2001) 12690-12691

39. T. Sanji, K. Takase and H. Sakurai, "The Induction of a Helical Conformation in Polysilanes with an Optically Active Terminal Group,” Bull Chem. Soc. Jpn. 77 (2004) 1607-1611

40. S. J. Holder, M Achilleos and R. G. Jones, "Increasing Molecular Weight Parameters of a Helical Polymer through Polymerization in a Chiral Solvent," J. Am. Chem. Soc. 128 (2006) 12418-12419

41. P. Dellaportas, R. G. Jones and S. J. Holder, “Induction of Preferential Helical Screw Senses in Optically Inactive Polysilanes via Chiral Solvation,” Macromol. Rapid Commun. 23 (2002) 99-103

42. H. Nakashima and M. Fujiki, “Precise Control of Optical Properties and Global Conformations by Marked Substituent Effects in Poly(alkyl(methoxyphenyl)silane) Homoand Copolymers, " Macromolecules, 34 (2001) 7558-7564 
43. S. Nespurek, A. Kadashchuk, Yu. Skryshevski, A. Fujii and K. Yoshino, “Origin of Broad Visible Luminescence in Poly[methyl(phenyl)silylene] Thin Films,” J. of Luminescence, 99 (2002) 131-140

44. O. Ito, M. Terajima, T. Azumi, N. Matsumoto, K. Takeda, M. Fujino and T. Azumi "The Photoluminescence of Poly(methylphenylsilylene): The Origin of the Long-wavelength Broad Band,” Macromolecules, 22 (1989)1709-1718 